Pacific

Journal of

Mathematics

RIEMANN MAPPING THEOREMS FOR BELTRAMI EQUATIONS BY CIRCLE PACKINGS

SHI-Yi LAN AND DAO-QING DAI

Volume $217 \quad$ No. 1

November 2004 


\title{
RIEMANN MAPPING THEOREMS FOR BELTRAMI EQUATIONS BY CIRCLE PACKINGS
}

\author{
SHI-YI LAN AND DAO-QING DAI
}

\begin{abstract}
We use circle packing techniques to construct approximate solutions to the generalized Beltrami equations with simply and multiply connected regions in the plane. We show convergence of the approximate solutions. This gives a constructive proof for the existence of quasiconformal mappings with a given pair of complex dilations.
\end{abstract}

\section{Introduction}

Circle packings - configurations of circles with specified patterns of tangency and having disjoint interiors - came to prominence with analysts in 1985 when Thurston conjectured that maps between such configurations would approximate conformal maps. Shortly, Burt Rodin and Dennis Sullivan [7] proved the convergence of Thurston's scheme, and much research on circle packings followed.

In [1] , Z.-X. He used circle packing methods to solve the Beltrami equation

$$
\partial_{\bar{z}} f=\lambda \partial_{z} f .
$$

In this paper we shall construct homeomorphic solutions $w=f(z)$ of the generalized Beltrami equation

$$
\partial_{\bar{z}} f=\lambda \partial_{z} f+\mu \overline{\partial_{z} f}, \quad z \in \Omega .
$$

We suppose that $\Omega$ is a simply or multiply connected Jordan domain in the complex plane $\mathbb{C}$ and that $\mathbb{U}$ is either, as the corresponding case may be, the unit disk with center at the origin or the unit circular domain obtained from the unit disk by the deletion of a closed disjoint union of finitely many closed disks and points. Further we assume that $\lambda, \mu: \Omega \rightarrow \mathbb{C}$ are measurable functions with

$$
\||\lambda|+|\mu|\|_{\infty}=\operatorname{esssup}_{z \in \Omega}(|\lambda|+|\mu|)<1 .
$$

The homeomorphic solution in $L_{2}$ to (1) and (2) is called a quasiconformal mapping with complex dilations $(\lambda, \mu)$.

This paper can be viewed as a development of [1] in terms of both equation and region. Our method of constructing functions is different from that of [1], and avoids an essential difficulty that one is faced with in discussing the Riemann's existence theorem of Equation (1): namely, the composition of a 
quasiconformal map with complex dilations $(\lambda, \mu)$ and a conformal map is a quasiconformal map, but its complex dilations are different from $(\lambda, \mu)$ (see [5], for example).

\section{Construction of Approximate Solutions}

We shall construct approximate solutions of Equation (1) in this section.

Let $\delta$ be a small positive number. We use the grid

$$
\mathrm{RL}(\delta)=\left\{x+i y: x=p \delta, y=\frac{\sqrt{3}}{2} q \delta,(p, q) \in \mathbb{Z} \times \mathbb{Z}\right\}
$$

to decompose $\mathbb{C}$ into a number of rectangles with side lengths $\delta$ and $\sqrt{3} \delta / 2$, and choose the side lengths of the rectangles in such a way that one is just able to use the regular hexagonal grid of mesh $\delta / n(n \in \mathbb{N})$ to triangulate it. Suppose $\bar{\Omega}_{\delta}$ is the minimal Jordan domain containing all rectangles that intersect $\Omega$. Then $\bar{\Omega}_{\delta}$ is a closed polyhedral Jordan domain, which is the union of squares of side length $\delta$ and $\sqrt{3} \delta / 2$. It is obvious that $\bar{\Omega} \subset \bar{\Omega}_{\delta}$ and $\bar{\Omega}_{\delta}$ converges to $\bar{\Omega}$ when $\delta \rightarrow 0$ in the sense that any compact subset of $\hat{\mathbb{C}} \backslash \bar{\Omega}$ is contained in $\hat{\mathbb{C}} \backslash \bar{\Omega}_{\delta}$ for $\delta$ small enough.

For any rectangle $R$ in $\bar{\Omega}_{\delta}$, set

$$
\begin{aligned}
& \lambda(R)= \begin{cases}\frac{1}{A(R)} \iint_{R} \lambda(z) d x d y, & R \subset \Omega, \\
0, & \text { otherwise, }\end{cases} \\
& \mu(R)= \begin{cases}\frac{1}{A(R)} \iint_{R} \mu(z) d x d y, & R \subset \Omega, \\
0, & \text { otherwise, }\end{cases}
\end{aligned}
$$

where $A(R)$ denotes the area of $R$. Define piecewise constant functions $\lambda_{\delta}, \mu_{\delta}: \Omega \rightarrow \mathbb{C}$ by

$$
\lambda_{\delta}(z)=\lambda(R), \quad \mu_{\delta}(z)=\mu(R), \quad \text { a.e. } z \in R \cap \Omega .
$$

Then

$$
\lim _{\delta \rightarrow 0} \lambda_{\delta}(z)=\lambda(z), \quad \lim _{\delta \rightarrow 0} \mu_{\delta}(z)=\mu(z), \quad \text { a.e. } z \in \Omega .
$$

Suppose that $R$ is an interior rectangle in $\bar{\Omega}_{\delta}$ with $R \cap \partial \bar{\Omega}_{\delta}=\emptyset$. Denote by $z_{1}, z_{2}, z_{3}$ and $z_{4}$ its lower left, lower right, upper right, and upper left vertices, respectively. Let $\Psi_{R}: \mathbb{C} \rightarrow \mathbb{C}$ be the map defined by

$$
\Psi_{R}(z)=\frac{|1+\lambda(R)+\mu(R)|}{1-|\lambda(R)+\mu(R)|^{2}}\left(\left(z-z_{1}\right)+(\lambda(R)+\mu(R))\left(\bar{z}-\bar{z}_{1}\right)\right) .
$$

It is easy to verify that $\partial_{\bar{z}} \Psi_{R}(z)=\lambda(R) \partial_{z} \Psi_{R}(z)+\mu(R) \overline{\partial_{z} \Psi_{R}(z)}$. Since $|\lambda(R)+\mu(R)| \leq\||\lambda|+|\mu|\|_{\infty}<1$, the function $\Psi_{R}(z)$ is an affine conformal homeomorphism with complex dilations $(\lambda(R), \mu(R))$ and maps the square $R$ 
onto the parallelogram $R^{\prime}$ with vertices $z_{1}^{\prime}=\Psi_{R}\left(z_{1}\right)=0, z_{2}^{\prime}=\Psi_{R}\left(z_{2}\right)=a$, $z_{3}^{\prime}=\Psi_{R}\left(z_{3}\right)=a+b, z_{4}^{\prime}=\Psi_{R}\left(z_{4}\right)=b$, where

$$
\begin{aligned}
& a=\frac{|1+\lambda(R)+\mu(R)|}{1-|\lambda(R)+\mu(R)|^{2}} \delta(1+\lambda(R)+\mu(R)), \\
& b=\frac{|1+\lambda(R)+\mu(R)|}{2\left(1-|\lambda(R)+\mu(R)|^{2}\right)} \sqrt{3} \delta(1-(\lambda(R)+\mu(R))) i .
\end{aligned}
$$

Write $n=2[1 /(2 \delta)], \omega=e^{i \pi / 3}$ and $\gamma=\delta / n$. Let $\operatorname{HL}(\gamma)$ denote the regular hexagonal grid with mesh $\gamma$, whose vertices form the lattice $V(\gamma)=$ $\{\gamma p+\gamma q \omega: p, q \in \mathbb{Z}\}$. The neighbors of $\alpha \in V(\gamma)$ are the points $\alpha+\gamma \omega^{k}$, $0 \leq k \leq 5$. Suppose $T(\gamma)$ is the complex obtained by using $\operatorname{HL}(\gamma)$ to triangulate the plane $\mathbb{C}$. Then the 1 -skeleton of $T(\gamma)$ is the nerve of some regular hexagonal circle packing $P_{\gamma}$ of the complex plane $\mathbb{C}$ formed by closed disks of radii $\gamma / 2$.

By (5), (6) and the fact that $|\lambda(R)+\mu(R)| \leq\||\lambda|+|\mu|\|_{\infty}<1$, it is easy to deduce that the angle $z_{4}^{\prime} z_{1}^{\prime} z_{2}^{\prime}$ of the parallelogram $R^{\prime}$ is bounded from below, the distance between $z_{1}^{\prime} z_{2}^{\prime}$ and $z_{4}^{\prime} z_{3}^{\prime}$ is $\sqrt{3} \delta / 2$ and the lengths of sides of $R^{\prime}$ are between $c \delta$ and $K \delta$, where $0<c \leq 1$ and $K \geq 1$ are constants depending only on $\||\lambda|+|\mu|\|_{\infty}$. So one can suppose that $H\left(R^{\prime}\right)$ is the collection of all triangles of $T^{(r)}(\gamma)$ lying in $R^{\prime}$ and having distance at least $\gamma / 2$ from $\partial R^{\prime}$, where $T^{(r)}(\gamma)=e^{i \theta} T(\gamma)$, for $\theta=\arg (1+\lambda(R)+\mu(R))$. Thus $H\left(R^{\prime}\right)$ is a simplicial complex and $\left|H\left(R^{\prime}\right)\right|$ is some polyhedral Jordan domain in $R^{\prime}$.

Since triangles in $H\left(R^{\prime}\right)$ have length $\delta / n$ one gets, as in [2], a triangulation $T\left(R^{\prime}\right)$ on $R^{\prime}$ that agrees with the triangulation $H\left(R^{\prime}\right)$ on $\left|H\left(R^{\prime}\right)\right|$ and whose restriction on each side of $R^{\prime}$ is the triangulation consisting of $n 1$-simplexes of equal length, the length lying between $c \delta / n$ and $K \delta / n$.

Set $(T(R), t)=\left(T\left(R^{\prime}\right),\left(\Psi_{R}\right)^{-1}\right)$. Then $(T(R), t)$ is a $K$-quasiconformal affine triangulation on the rectangle $R$, and its restriction on each side of $R$ is the affine triangulation with $n 1$-simplexes of equal length. So these affine triangulations can be glued together to form a $K$-quasiconformal affine triangulation for

$$
\widetilde{\Omega}_{\delta}=\bigcup_{R \cap \partial \bar{\Omega}=\phi} R .
$$

In addition, by intersecting the region

$$
\bar{\Omega}_{\delta} \backslash \operatorname{int} \widetilde{\Omega}_{\delta}=\bigcup_{R \cap \partial \bar{\Omega}_{\delta \neq \phi}} R
$$

with the regular hexagonal triangulation $\operatorname{HL}(\gamma)$ of the plane, one gets an affine triangulation $\left(\widetilde{T}_{\delta}, I\right)$ for $\bar{\Omega}_{\delta} \backslash$ int $\widetilde{\Omega}_{\delta}$, where $I$ denotes the identity map. 
Pasting together the affine triangulations above for $\widetilde{\Omega}_{\delta}$ and $\bar{\Omega}_{\delta} \backslash$ int $\widetilde{\Omega}_{\delta}$, we obtain an affine triangulation $\left(T_{\delta}, t_{\delta}\right)$ for $\bar{\Omega}_{\delta}$.

Denote by $L_{m}(R)$ the subcomplex of $H(R)=\Psi_{R}^{-1}\left(H\left(R^{\prime}\right)\right)$ consisting of faces of triangles with the property that $G\left(T_{\delta}, v, m\right) \subset H(R)$ for each vertex $v$ of $\Delta$, where $G\left(T_{\delta}, v, m\right)$ is $m$ generations about $v$ in the complex $T_{\delta}$. From the preceding discussion, it is not difficult to obtain:

\section{Lemma 1.}

(a) For $\widetilde{\Omega}_{\delta}=\bigcup_{R \cap \partial \bar{\Omega}=\phi} R$, one has $\lim _{\delta \rightarrow 0} A\left(\Omega \backslash \widetilde{\Omega}_{\delta}\right)=0$.

(b) Let $m$ be a nonnegative integer with $m \leq c n$, for any square $R$ in $\widetilde{\Omega}_{\delta}$. Then

$$
A\left(\left|H(R) \backslash L_{m}(R)\right|\right) \leq c A(R)(m+1) / n,
$$

where $A$ denotes area.

In addition, for the hexagonal circle packing, we have:

Lemma 2. Let $H_{n}$ be the n-generation of a circle packing $P$ about some circle $c_{0}$ of $P$ such that $H_{n}$ is combinatorially equivalent to $H_{n}^{\prime}$, where $H_{n}^{\prime}$ is the n-generation of some regular hexagonal circle packing about one of its circles, say $c_{0}^{\prime}$. For $n$ large enough, there is a quasiconformal mapping $\Phi$ from the complex plane $\mathbb{C}$ to $\mathbb{C}$ that maps the subpacking $H_{m}^{\prime}$ of $H_{n}^{\prime}$ (with $m \sim n / 2$ ) to the corresponding subpacking $H_{m}$ of $H_{n}$ and whose restriction $\left.\Phi\right|_{D_{0}^{\prime}}$ to the closure $D_{0}^{\prime}$ of the interior of $c_{0}^{\prime}$ is Lipschitz and can be expressed by $\left.\Phi\right|_{D_{0}^{\prime}}=\left(r / r^{\prime}\left(z-a_{0}^{\prime}\right)+a_{0}\right)+h(z) / n$, where $a_{0}, a_{0}^{\prime}, r, r^{\prime}$ are the centers and radii of $c_{0}, c_{0}^{\prime}$, and $|h(z)|$ is bounded above by a positive constant $M$.

Proof. By the transformation $\zeta=m(z)=r / r^{\prime}\left(z-a_{0}^{\prime}\right)+a_{0}, H_{n}^{\prime}$ becomes $H_{n}^{\prime \prime}$. It is clear that $H_{n}^{\prime \prime}$ is combinatorially equivalent to $H_{n}^{\prime}$ and $c_{0}^{\prime \prime}=c_{0}$, where $c_{0}^{\prime \prime}=m\left(c_{0}^{\prime}\right)$. Since $H_{n}^{\prime}$ is combinatorially equivalent to $H_{n}$, so is $H_{n}^{\prime \prime}$. We now construct the quasiconformal mapping $\Phi$. Fix any three mutually tangent circles $c_{1}^{\prime \prime}, c_{2}^{\prime \prime}, c_{3}^{\prime \prime}$ in $H_{n-1}^{\prime \prime}$ and the corresponding circles $c_{1}, c_{2}, c_{3}$ in $H_{n-1}$. Let $\varphi$ be the orientation-preserving Möbius transformation sending $c_{j}^{\prime \prime} \cap c_{k}^{\prime \prime}$ to $c_{j} \cap c_{k}$, where $(j, k)$ is any pair in $\{(1,2),(1,3),(2,3)\}$. Obviously, $\varphi$ maps the interstice bounded by $c_{1}^{\prime \prime}, c_{2}^{\prime \prime}$ and $c_{3}^{\prime \prime}$ to that bounded by $c_{1}, c_{2}$ and $c_{3}$. These conformal mappings may be glued together to form a conformal mapping from the union of interstice bounded by circles of $H_{n-1}^{\prime \prime}$ to the union of interstice bounded by circles of $H_{n-1}$. The mapping maps each circle of $H_{n-2}^{\prime \prime}$ to the corresponding circle of $H_{n-2}$. Next, we extend the mapping radially on each disk bounded by circles of $H_{n-2}^{\prime \prime}$ and the result is a $K$ quasiconformal mapping $\varphi_{1}$ from the union of interstices and disks bounded by circles of $H_{n-2}^{\prime \prime}$ to the corresponding union bounded by circles of $H_{n-2}$. Finally, by a property of quasiconformal mappings, $\varphi_{1}$ can be extended to a $K_{1}$-quasiconformal mapping $\Phi_{1}: \hat{\mathbb{C}} \rightarrow \hat{\mathbb{C}}$ with $\Phi_{1}(\infty)=\infty$ such that $\Phi_{1}$ 
equals $\varphi_{1}$ on the union of interstice and the disks bounded by circles of $H_{m}^{\prime \prime}$, where $m=[(n-3) / 2]$. From [2], we obtain $\left.\Phi_{1}\right|_{D_{0}^{\prime \prime}}(\zeta)=\zeta+h_{1}(\zeta) / n$, where $\left|h_{1}(\zeta)\right| \leq M$ and $M$ is a positive constant. Let $\Phi(z)=\Phi_{1}(m(z))$; then $\left.\Phi\right|_{D_{0}^{\prime}}=\left(r / r^{\prime}\left(z-a_{0}^{\prime}\right)+a_{0}\right)+h(z) / n$, where $h(z)=h_{1}(m(z))$. From the construction of $\Phi$, it is easy to get that $\Phi$ is Lipschitz on $D_{0}^{\prime}$. So $\Phi$ is the desired mapping.

According to the circle packing theorem [8], given an abstract triangulation $T$ of a topological sphere, there exists a circle packing $P$ on the Riemann sphere $\mathbb{S}^{2}$ having the combinatorics of $T . P$ is unique up to Möbius transformations of $\mathbb{S}^{2}$. We further obtain:

Lemma 3. Let $T$ be a simply or multiply connected complex in the plane. There exists a circle packing $P$ in the unit disk or some unit circle domain, as the case may be, whose tangency graph is isotopic to the 1-skeleton of $T$. Moreover, $P$ is unique up to Möbius transformation.

Proof. If $T$ is simply connected, the conclusion of the lemma holds from [7]. If $T$ is a multiply connected complex, by adding an ideal vertex for each hole and connecting each ideal vertex and boundary vertices of $T$ neighboring it with disjoint Jordan arcs, we get the augmented complex $T^{*}$, which is simply connected. By the result in the simply connected case, there exists a circle packing $P^{*}$ in the unit disk whose tangency graph is isomorphic to the 1-skeleton of $T^{*}$. By removing the circles corresponding to the ideal vertices in $P^{*}$, we obtain a circle packing $P$ in the unit circle domain with the combinatorics of $T$ and $P$ is unique up to Möbius transformation.

For the affine triangulation $\left(T_{\delta}, t_{\delta}\right)$ of $\bar{\Omega}_{\delta}$, by Lemma 3 , there is a circle packing $P_{\delta}$ in the closed region $\overline{\mathbb{U}}$, which has the combinatorics of $T_{\delta}$ and is unique up to Möbius transformation of the unit disk, where $\mathbb{U}$ is the unit disk or the unit circle domain.

We construct approximate solutions of Equation (1) as follows:

(a) For any $\left|L_{m}\left(R^{\prime}\right)\right| \subset\left|T_{\delta}\right|$, with $L_{m}\left(R^{\prime}\right)=\Psi_{R}\left(L_{m}(R)\right)$, let $\zeta_{0}$ be any vertex of $L_{m}\left(R^{\prime}\right)$. There is an $m$-generation of some regular hexagonal circle packing about one of its circles, say, $c_{0}$ of center $\zeta_{0}$ and radius $\delta /(2 n)$, which is combinatorially equivalent to some $m$-generation of the circle packing $P_{\delta}$ about one of its circle, say $c_{0}^{\prime}$, in $\overline{\mathbb{U}}$. We define $g_{\delta}: D_{0} \rightarrow D_{0}^{\prime}$ by $g_{\delta}=\left.\Phi\right|_{D_{0}}$, where $\Phi$ is as in Lemma 2 and $D_{0}, D_{0}^{\prime}$ are regions bounded by $c_{0}$ and $c_{0}^{\prime}$. By pasting these mappings, we obtain a quasiconformal mapping from the union of disks bounded by the circles whose centers are the vertices of $L_{m}\left(R^{\prime}\right)$ and whose radii are all equal to $\delta /(2 n)$ to the corresponding union of bounded circles of $P_{\delta}$ in $\overline{\mathbb{U}}$. We then extend linearly the mapping to the interstices bounded by these circles and the result is a $K$-quasiconformal mapping $G_{\delta}$ from the union of interstices and disks bounded by circles whose centers 
are the vertices of $L_{m}\left(R^{\prime}\right)$ and whose radii are all equal to $\delta /(2 n)$ to the corresponding union of bounded by circles of $P_{\delta}$ in $\overline{\mathbb{U}}$. Let $\widetilde{G_{\delta}}=\left.G_{\delta}\right|_{\left|L_{m}\left(R^{\prime}\right)\right|}$. Then $\widetilde{G_{\delta}}$ is a $K$-quasiconformal mapping from $\left|L_{m}\left(R^{\prime}\right)\right|$ to the corresponding portion of $\left|\operatorname{carr}\left(P_{\delta}\right)\right|$, where $\operatorname{carr}\left(P_{\delta}\right)$ denotes the concrete geometric complex in $\overline{\mathbb{U}}$ formed by connecting the centers of tangent circles of $P_{\delta}$ with line segments.

(b) For $\left|T_{\delta}\right| \backslash\left(\bigcup_{R \cap \partial \bar{\Omega}=\phi}\left|L_{m}(R)\right|\right)$, let $\hat{G}_{\delta}$ be the map sending any vertex of $T_{\delta} \backslash\left(\bigcup_{R_{\delta} \cap \partial \bar{\Omega}=\phi} L_{m}\left(R_{\delta}\right)\right)$ to the center of the corresponding disk and then extend it linearly on each simplex of $T_{\delta} \backslash\left(\bigcup_{R_{\delta} \cap \partial \bar{\Omega}=\phi} L_{m}\left(R_{\delta}\right)\right)$. Then $\hat{G}_{\delta}$ is a $K$-quasiconformal mapping from $\left|T_{\delta}\right| \backslash\left(\bigcup_{R_{\cap} \partial \bar{\Omega}=\phi}\left|L_{m}\left(R_{\delta}\right)\right|\right)$ to the corresponding portion of $\left|\operatorname{carr}\left(P_{\delta}\right)\right|$.

(c) By pasting $\hat{G}_{\delta}$ and the $\widetilde{G_{\delta}}$, we get a $K$-quasiconformal mapping $H_{\delta}$ from $\left|T_{\delta}\right|$ to $\left|\operatorname{carr}\left(P_{\delta}\right)\right| \subset \overline{\mathbb{U}}$. Let $F_{\delta}=H_{\delta} \circ t_{\delta}^{-1}$, then $F_{\delta}$ is a homeomorphic mapping from $\bar{\Omega}_{\delta}$ to $\left|\operatorname{carr}\left(P_{\delta}\right)\right| \subset \overline{\mathbb{U}}$.

We next prove that $f_{\delta}$ converges to some solution of the generalized Beltrami equation (1) as $\delta$ approaches 0 .

\section{Convergence of approximate solutions}

In this section, we show the existence of homeomorphic solutions for the generalized Beltrami equation (1).

Theorem 1. Let $\lambda, \mu$ be two measurable functions with $\||\lambda|+|\mu|\|_{\infty}<1$. For any positive sequence $\delta_{k}$ with $\delta_{k} \rightarrow 0$, let $f_{\delta_{k}}: \Omega \rightarrow \mathbb{U}$ be the sequence of map constructed for each $\delta_{k}$ through the procedure in Section 2. Then $f_{\delta_{k}}$ has a subsequence converging uniformly on every compact subset of $\Omega$ to some quasiconformal homeomorphism $f: \Omega \rightarrow \mathbb{U}$ that solves the generalized Beltrami equation

$$
\partial_{\bar{z}} f(z)=\lambda \partial_{z} f(z)+\mu \overline{\partial_{z} f(z)}, \quad \text { a.e. } z \in \Omega .
$$

Proof. First, $t_{\delta}^{-1}: \bar{\Omega}_{\delta} \rightarrow\left|T_{\delta}\right|$ is an affine map, and from its construction we see that $F_{\delta}=H_{\delta} \circ t_{\delta}^{-1}: \bar{\Omega}_{\delta} \rightarrow\left|\operatorname{carr}\left(P_{\delta}\right)\right|$ is a $K$-quasiconformal homeomorphism. Set $\widetilde{f}_{\delta_{k}}=F_{\delta_{k}}=H_{\delta_{k}} \circ t_{\delta_{k}}^{-1}$, where $\delta_{k} \rightarrow 0$. Then $f_{\delta_{k}}=\left.\widetilde{f}_{\delta_{k}}\right|_{\Omega}: \Omega \rightarrow \mathbb{U}$ is $K$-quasiconformal, too. Based on the convergence theorem for quasiconformal maps (see [4] or [5]), there is a subsequence of $f_{\delta_{k}}$, still denoted by $f_{\delta_{k}}$, that converges uniformly on every compact subset of $\Omega$ to $f$, where $f$ is some quasiconformal homeomorphism from $\Omega$ to $\mathbb{U}$.

Next we show that $f$ has complex dilations $(\lambda, \mu)$. Suppose that $m_{k}$ is a sequence of positive integer such that $m_{k} \rightarrow \infty$ and $m_{k} / n_{k} \rightarrow 0$. For any $z \in \sigma \subset\left|L_{m_{k}}(R)\right|$, by the definition of $f_{\delta_{k}}$, we have $f_{\delta_{k}}(z)=H_{\delta_{k}} \circ t_{\delta_{k}}^{-1}(z)=$ $H_{\delta_{k}} \circ \Psi_{R}(z)$. There are two situations for $\Psi_{R}(z)$ : 
(i) $\Psi_{R}(z)$ lies in some disk $D_{0}$ of center $\zeta_{0}$ and radius $r_{0}=\delta_{k} /\left(2 n_{k}\right)$. Then

$$
f_{\delta_{k}}(z)=\left(r_{0}^{\prime} / r_{0}\left(\Psi_{R}(z)-\zeta_{0}\right)+w_{0}\right)+h\left(\Psi_{R}(z)\right) / m_{k},
$$

where $w_{0}$ and $r_{0}^{\prime}$ are the center and radius of the corresponding circle in $P_{\delta_{k}}$. Taking partial derivatives of $f_{\delta_{k}}$, we get

$$
\begin{aligned}
& \partial_{\bar{z}} f_{\delta_{k}}(z)=r_{0}^{\prime} / r_{0} \partial_{\bar{z}} \Psi_{R}(z)+\partial_{\bar{z}} h\left(\Psi_{R}(z)\right) / m_{k}, \\
& \partial_{z} f_{\delta_{k}}(z)=r_{0}^{\prime} / r_{0} \partial_{z} \Psi_{R}(z)+\partial_{z} h\left(\Psi_{R}(z)\right) / m_{k} .
\end{aligned}
$$

Since $\partial_{\bar{z}} \Psi_{R}(z)=\lambda_{\delta_{k}} \partial_{z} \Psi_{R}(z)+\mu_{\delta_{k}} \overline{\partial_{z} \Psi_{R}(z)}$, we have

$$
\begin{aligned}
\partial_{\bar{z}} f_{\delta_{k}}(z)=\lambda_{\delta_{k}} \partial_{z} f_{\delta_{k}}(z)+\mu_{\delta_{k}} \overline{\partial_{z} f_{\delta_{k}}(z)} & \\
& +\left(\partial_{\bar{z}} h\left(\Psi_{R}(z)\right) / m_{k}+2 \operatorname{Re}\left(\partial_{z} h\left(\Psi_{R}(z)\right)\right) / m_{k}\right) ;
\end{aligned}
$$

by Lemma 2 and the definition of $\Psi_{R}(z)$, we conclude that $\partial_{\bar{z}} h\left(\Psi_{R}(z)\right)$ and $\partial_{z} h\left(\Psi_{R}(z)\right)$ are bounded on $L_{m_{k}}(R)$. Combining with (4) and letting $k \rightarrow \infty$, because $r_{0}^{\prime} / r_{0}$ converges to the modulus of the derivative of some Riemann mapping function $[6]$, we obtain from (7)

$$
\partial_{\bar{z}} f(z)=\lambda \partial_{z} f(z)+\mu \overline{\partial_{z} f(z)} .
$$

(ii) $\Psi_{R}(z)$ lies in the interstice bounded by three circles $c_{0}, c_{1}$ and $c_{2}$. Then $\Psi_{R}(z)=(1-t) \zeta^{\prime}+t \zeta^{\prime \prime}$, where $0 \leq t \leq 1, \zeta^{\prime} \in c_{0}$, and $\zeta^{\prime \prime} \in c_{l}$, for $l=1$ or $l=2$. Write $\zeta_{j}$ and $r_{j}(j=0,1,2)$ for the center and radius of $c_{j}$. It follows from the definition of $f_{\delta_{k}}(z)$ that

$$
\begin{aligned}
f_{\delta_{k}}(z)= & (1-t)\left(\left(r_{0}^{\prime} / r_{0}\left(\zeta^{\prime}-\zeta_{0}\right)+w_{0}\right)+h\left(\zeta^{\prime}\right) / m_{k}\right) \\
& +t\left(\left(r_{l}^{\prime} / r_{l}\left(\zeta^{\prime}-\zeta_{l}\right)+w_{l}\right)+h\left(\zeta^{\prime \prime}\right) / m_{k}\right) \\
= & r_{0}^{\prime} / r_{0} \Psi_{R}(z)+\left(r_{l}^{\prime}-r_{0}^{\prime}\right) / r_{l} q\left(\Psi_{R}(z)\right)+\widetilde{q}\left(\Psi_{R}(z)\right)+C,
\end{aligned}
$$

where

$$
\begin{aligned}
q\left(\Psi_{R}(z)\right)=t \zeta^{\prime \prime} & =\Psi_{R}(z)-(1-t) \zeta^{\prime}, \widetilde{q}\left(\Psi_{R}(z)\right)=(1-t) h\left(\zeta^{\prime}\right)+t h\left(\zeta^{\prime \prime}\right), \\
C & =(1-t)\left(w_{0}-r_{0}^{\prime} / r_{0} \zeta_{0}\right)+t\left(w_{j}-r_{l}^{\prime} / r_{l} \zeta_{l}\right)
\end{aligned}
$$

and $w_{j}$ and $r_{j}^{\prime}(j=0,1,2)$ are the center and radius of the corresponding circle. By [6] and [2], we have $\left(r_{l}^{\prime}-r_{0}^{\prime}\right) / r_{l}=r_{0}^{\prime} / r_{l} O\left(1 / m_{k}\right)$, and note that $\partial_{\bar{z}} q\left(\Psi_{R}(z)\right), \partial_{z} q\left(\Psi_{R}(z)\right), \partial_{\bar{z}} \widetilde{q}\left(\Psi_{R}(z)\right)$ and $\partial_{z} \widetilde{q}\left(\Psi_{R}(z)\right)$ are bounded on $\left|L_{m_{k}}(R)\right|$. As in (i), it follows from (8) that

$$
\partial_{\bar{z}} f(z)=\lambda \partial_{z} f(z)+\mu \overline{\partial_{z} f(z)}, \quad k \rightarrow \infty .
$$

Thus, for any $R \subset \widetilde{\Omega}_{\delta_{k}}$, we obtain from (i) and (ii) that

$$
\partial_{\bar{z}} f(z)=\lambda \partial_{z} f(z)+\mu \overline{\partial_{z} f(z)}, \quad z \in\left|L_{m_{k}}(R)\right|, k \rightarrow \infty .
$$


On the other hand, for any $z \in \Omega \backslash L_{k}$, where $L_{k}=\bigcup_{R \cap \partial \bar{\Omega}_{\delta_{k}}=\phi}\left|L_{m_{k}}(R)\right|$, we get easily from Lemma 1 that

$$
\lim _{k \rightarrow \infty} A\left(\Omega \backslash L_{k}\right)=0 .
$$

Therefore, as $k \rightarrow \infty$, we conclude that

$$
\partial_{\bar{z}} f(z)=\lambda \partial_{z} f(z)+\mu \overline{\partial_{z} f(z)}, \quad \text { a.e. } z \in \Omega,
$$

that is, the complex dilations of the quasiconformal mapping $f$ are $(\lambda, \mu)$.

Theorem 2. The maps $f_{\delta}$ constructed in Section 2, as $\delta \rightarrow 0$, converge uniformly on each compact subset of $\Omega$ to a solution $f: \Omega \rightarrow \mathbb{U}$ of (1).

Proof. By their construction, the $f_{\delta}$ must converge to a homeomorphic map $\Omega \rightarrow \mathbb{U}$ as $\delta \rightarrow 0$. Using Theorem 1 , we can get that the $f_{\delta}$, as $\delta \rightarrow 0$, converge uniformly on every compact subset of $\Omega$ to some solution $f: \Omega \rightarrow \mathbb{U}$ of (1).

\section{References}

[1] Z.-X. He, Solving Beltrami equations by circle packings, Trans. Amer. Math. Soc., 322 (1990), 657-670, MR 0974518 (91c:30032), Zbl 0714.30012.

[2] Z.-X. He, An estimate for hexagonal circle packings, J. Differential Geom., 33 (1991), 395-412, MR 1094463 (92b:52039), Zbl 0743.52005.

[3] Z.-X. He and O. Schramn, The $C^{\infty}$-convergence of hexagonal disk packings to the Riemann map, Acta Math., 180 (1998), 219-245, MR 1638772 (99j:52021), Zbl 0913.30004.

[4] O. Lehto and K.I. Virtanen, Quasiconformal Mappings in the Plane (second ed.), Die Grundlehren der mathematischen Wissenschaften, 126, Springer-Verlag, New York, 1973, MR 0344463 (49 \#9202), Zbl 0267.30016.

[5] Z. Li, Quasiconformal Mapping and its Application to Riemann Surfaces, Science Press, Beijing, 1988 (in Chinese).

[6] B. Rodin, Schwarz's lemma for circle packings (II), J. Differential Geom., 30 (1989), 539-554, MR 1010171 (90m:11099), Zbl 0646.30014.

[7] B. Rodin and D. Sullivan, The convergence of circle packings to the Riemann mapping, J. Differential Geom., 26 (1987), 349-360, MR 0906396 (90c:30007), Zbl 0694.30006.

[8] W. Thurston, The Geometry and Topology of 3-Manifolds, Princeton University Notes, Princeton, NJ, 1979.

[9] G.B. Williams, Approximation of quasisymmetries using circle packings, Discrete Comput. Geom., 25 (2001), 103-124, MR 1797299 (2002h:52033), Zbl 0984.30028.

Received December 15, 2003 and revised February 20, 2004. This work is supported in part by NSF of China and grants from the Ministry of Education of China.

Department of Mathematics

Sun Yat-Sen (Zhongshan) University

Guangzhou, 510275, P.R. China 
Department of Mathematics

Guangxi University for Nationalities

NANNING, 530006

P.R. ChinA

E-mail address: shiyilan06@sohu.com

Department of Mathematics

Sun Yat-Sen (Zhongshan) University

Guangzhou, 510275

P.R. CHINA

E-mail address: stsddq@zsu.edu.cn 